Material Science and Engineering with Advanced Research

\title{
Comparative Study of Hemostatic Agents Using Thrombelastography
}

\author{
Henry T. Peng \\ Defence Research and Development Canada, Toronto Research Centre, 1133 Sheppard Avenue West, Toronto, Ontario, Canada M3K 2C9
}

*Corresponding author: Henry T. Peng, Defence Research and Development Canada, Toronto Research Centre, 1133 Sheppard Avenue West, Toronto, Ontario, Canada M3K 2C9; Tel: 01-4166352129; E mail: henry.peng@drdc-rddc.gc.ca

Article Type: Research, Submission Date: 29 April 2016, Accepted Date: 20 May 2016, Published Date: 13 June 2016.

Citation: Henry T. Peng (2016) Comparative Study of Hemostatic Agents Using Thrombelastography. Mater. Sci. Eng. Adv. Res 1(4) : 1-7. doi: https://doi.org/10.24218/msear.2015.16.

Copyright: @ 2016 Henry T. Peng. This is an open-access article distributed under the terms of the Creative Commons Attribution License, which permits unrestricted use, distribution, and reproduction in any medium, provided the original author and source are credited.

\begin{abstract}
Hemorrhage is one of the leading causes of death in civilian and military trauma. Thrombelastography (TEG) quantitatively measures the viscoelastic changes of whole blood during clotting, from the beginning of coagulation to the end with fibrinolysis. In this study, we successfully developedand used a TEG method to compare a well-known Chinese herb medicine (i.e., Yunnan Baiyao) for hemorrhage control with other commercial hemostatic products (e.g., Celox ${ }^{\mathrm{TM}}$ and QuikClot ${ }^{\mathrm{TM}}$ ) and chitosan in the form of dry powder or dispersion. When tested in the powder form, the herbal material outperformed Celox ${ }^{\mathrm{TM}}$ as indicated by all three TEG parameters (i.e., reaction time R, clot formation rate $\alpha$ and maximum clot strength MA), and was comparable with QuikClot ${ }^{\mathrm{TM}}$, but showed no exothermic effects. When tested in the dispersion form, which may be easier to use (as a spray) than in the powder form, the herbal material provided better hemostasis than the other materials, where the coagulation actions of the latter were reduced in the dispersion form. In addition, comparison among $\mathrm{Celox}^{\mathrm{TM}}$ and various types of chitosan showed the effects of physical and chemical properties of the chitosan-based biomaterials on hemostasis. The hemostatic mechanisms of these agents and their potential benefitsas an inexpensive, safe, and easily available material for hemorrhage control are discussed.
\end{abstract}

Keywords: Hemostatic agent, Thrombelastography, Hemorrhage control.

\section{Introduction}

Hemorrhage control is vital for clinical outcome after surgical treatment and prehospital trauma injuries. Both systemic and local strategies have been developed for management of surgical and traumatic bleeding. The former typically involves hemostatic resuscitation of blood products and coagulation factor concentrate [1], while the latter uses numerous hemostatic biomaterials [2]. Advances in hemostatic materials have been made in the past few years given the significant interests in hemorrhage control on the battlefield [3]. Chitosan-based Celox ${ }^{\mathrm{TM}}$ and HemCon ${ }^{\mathrm{TM}}$, and zeolite-based QuikClot ${ }^{\mathrm{TM}}$ are among the few most effective hemostatic products [4]. However, each product has their limitation due to either the side effects,e.g., thermal damage to the tissues by QuikClot ${ }^{\mathrm{TM}}$ [5] or the lack of efficacy for hemorrhage control in an injury with limited vessel access [4].

Yuannan Baiyao is a Chinese remedy clinically used for stopping bleeding from surgery [6], diseasessuch as advanced cancer and ulcer $[7,8]$ and combat trauma [9]. It has been administered both topically and orally. Given its other medical effects,e.g., antiinflammation, it has become a multipurpose remedy.

Thrombelastography (TEG) has been widely used in clinical settings for monitoring coagulopathy $[10,11]$ and guide hemostatic resuscitation in trauma [12]. In contrast, its use as a research tool for evaluating the effectiveness of hemostatic agents, especially those insoluble in blood, is limited [13].

In this study, we successfully applied a TEG method to test different hemostatic agents in the form of dry powder or dispersion. The ultimate objective is to develop a more effective, inexpensive, easy to store and use hemostatic agent.

\section{Materials and Methods}

\section{Hemostatic Agents}

Yunnan Baiyao was made by Yunnan Baiyao Group Co. Ltd (Yunnan, China). QuikClot ${ }^{\mathrm{TM}}$ was provided by Z-Medica Corporation (CT, USA). Celox ${ }^{\mathrm{TM}}$ was kindly provided by SAM Medical Products (TX, USA).Three types of medical grade chitosan derived from different natural resources were purchased. Chitosan derived from giant squid cartilage with a molecular weight larger than 1,000,000 and deacetylation degree higher than $90 \%$ in a power form was purchased from Arabio Co., Ltd. (Guui-Dong, Gwangin-Gu, Korea) for initial comparison with Yunnan Baiyao, QuikClot ${ }^{\mathrm{TM}}$ and Celox ${ }^{\mathrm{TM}}$. In addition, random and block chitosan was purchased from Biosyntech Canada Inc. (Laval, QC, Canada). Both were derived from crustacean shells, the former had a molecular weight of 223,000 and deacetylation degree of $95.1 \%$ and the latter had a molecular weight of 3,410,000 and deacetylation degree of $94.5 \%$. The hemostatic properties of the three types of chitosan were compared with the commercial products.

\section{Thrombelastography}

Figure 1(a) shows a $\mathrm{TEG}^{\mathrm{TM}} 5000$ hemostasis analyzer 


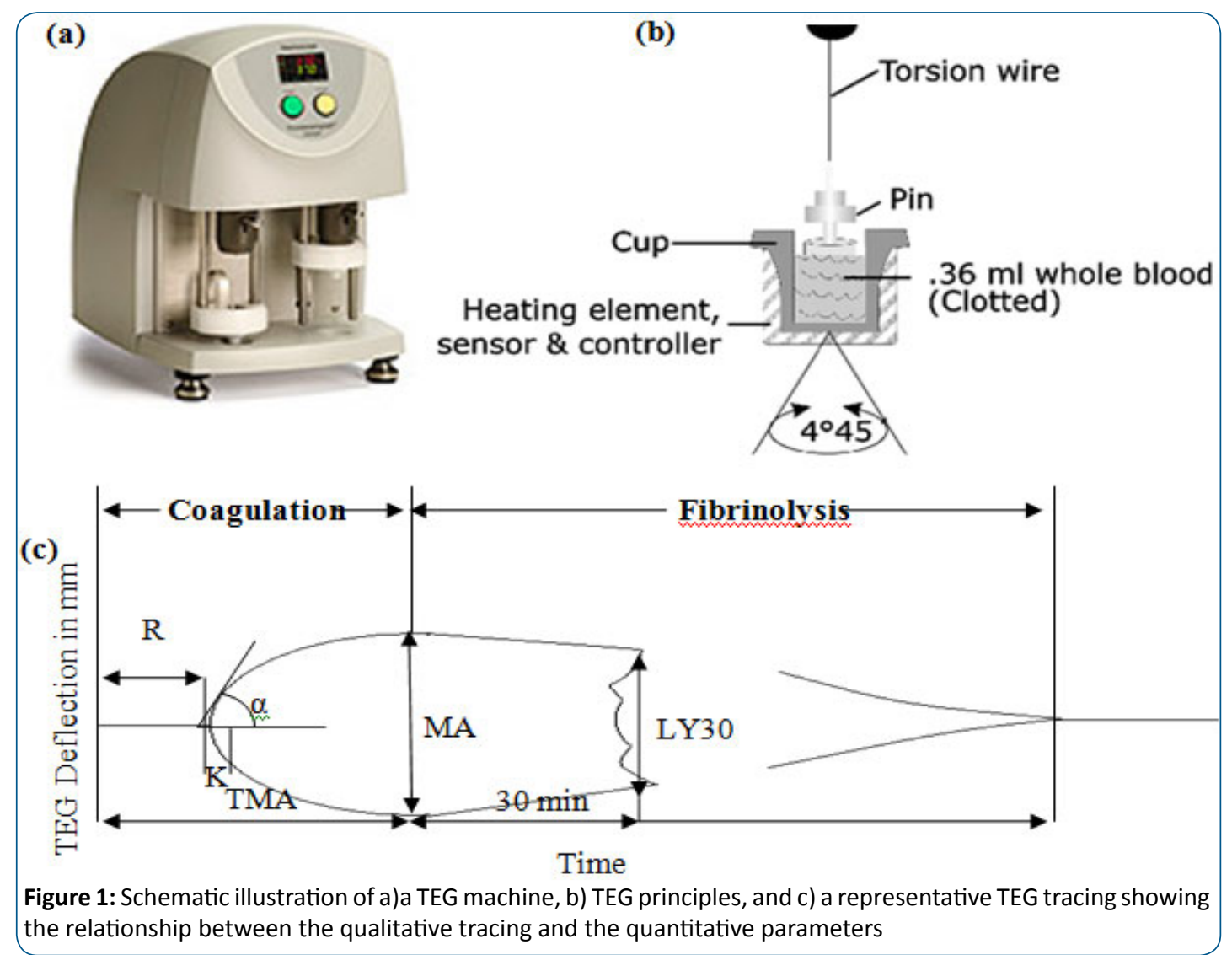

(Haemonetics Corporation, Haemoscope Division, Niles, IL, USA) and Figure 1(b) shows how it works. The machine measures the viscoelastic properties of blood as it clots under a low shear. It has two channels; for each channel, a pin suspended by a torsion wire is immersed in whole blood or plasma in a plastic cupmade of acrylic polymer with a smooth interior surface. The cup oscillates back and forth constantly at a set speed through an arc of $4^{\circ} 45^{\mathrm{TM}}$ every 5 seconds. The torque of the cup is transmitted to the pin, via the fibrin strands in the blood clots as coagulation proceeds, and to the torsion wire for conversion by a mechanical-electrical transducer to an electrical signal, which can be monitored by a computer [14]. The measurement is graphically represented as a characteristic shape profile over time (Figure 1c), from which the following parameters can be derived to provide main information about the coagulation and fibrinolysis: 1) reaction time ' $R$ ' or time to first clot formation, which is related to plasma clotting factors and circulating inhibitory activity; 2) coagulation time ' $\mathrm{K}$ ' or time to a specific level of clot strength, which is associated with the activity of the intrinsic clotting factors, fibrinogen and platelets; 3) rate of clot formation ' $\alpha$ angle' or rapidity of fibrin cross-linking, which is a main function of platelets, fibrinogen and plasma components residing on the platelet surface; 4) maximum amplitude 'MA' or maximum clot strength, which is a direct function of the maximum dynamic properties of fibrin and platelet number and function; 5) time to reach MA 'TMA'; and 6) fibrinolysis at $30 \mathrm{~min}$ 'LY30' or the rate of amplitude reduction $30 \mathrm{~min}$ after MA, which relates to fibrinolysis. In our study, three key TEG parameters were used for studying the effects of procoagulant agents [15]: 1) time to fibrin initiation (R); 2) rapidity of fibrin cross-linking $(\alpha)$; 3 ) maximum clot strength (MA).

To perform a TEG measurement, the analyzer was first calibrated through electronics testing and quality control according to manufacturer's protocol. A powder material at a various amount (0.5-2.0 mg) was mixed with 340- $\mu \mathrm{L}$ citrated human blood from healthy volunteers in a TEG sampling cup pre-warmed to $37^{\circ} \mathrm{C}$. Blood coagulation was initiated by adding $20 \mu \mathrm{L}$ of $0.2 \mathrm{M} \mathrm{CaCl}_{2}$ to the cup and the measurement was run immediately until all interested parameter values were obtained. The percentage difference for each parameter was calculated as follow: $\left(\mathrm{P}_{\mathrm{HA}}-\mathrm{P}_{0}\right) /$ $\mathrm{P}_{0}$, where $\mathrm{P}_{\mathrm{HA}}, \mathrm{P}_{0}$ are the parameters measured with or without addition of the hemostatic agent, respectively.+/- was used to denote either positive or negative effects on blood coagulation. For example, negative changes in $\mathrm{R}$ and positive changes in $\alpha$ and MA indicate an enhancement of clot formation and strength. The magnitude suggests the extent of the effects.

For the dispersion formulation, the material was suspended in a pH 7.4 Tris buffer and $20-\mu \mathrm{L}$ dispersion was mixed with $320-\mu \mathrm{L}$ citrated human blood from healthy volunteers in a TEG cup prewarmed to $37^{\circ} \mathrm{C}$, followed by adding $20 \mu \mathrm{L}$ of $0.2 \mathrm{M} \mathrm{CaCl}_{2}$. The percentage difference for each parameter was calculated using the above equation.

\section{Statistical Analysis}

Data were presented as mean \pm standard deviation and compared using a two-tailed $\mathrm{t}$ test with $95 \%$ confidence to identify significantly different groups.

\section{Results}

We confirmed that the TEG method is applicable in demonstrating the clotting effect of a known hemostatic agent, QuikClot ${ }^{\mathrm{TM}}[16]$, which we used as a positive control in this study (Figures 2-4). Figures 2-4 also showed the hemostatic properties of a first-aid herbal powder, YunanBaiyao. Specifically, the herbal material, 
when added as a powder in the amount of 0.5 to $2 \mathrm{mg}$, reduced $\mathrm{R}$ by $50-70 \%$ (Figure 2), increased $a$ by $20-60 \%$ (Figure 3 ), but had a minimal effect on MA (Figure 4). In contrast, QuikClot ${ }^{\mathrm{TM}}$ reduced $\mathrm{R}$ by $40-50 \%$, increased both $\alpha$ and MA by $30-100 \%$ and $7-20 \%$, respectively. Celox ${ }^{\mathrm{TM}}$ and chitosan led to less decrease in $\mathrm{R}$ ( -6 to $5 \%$ and -1 to $-40 \%)$, less increase in a (-4 to $36 \%$ and 0.6 to $78 \%)$, and $\mathrm{MA}(0.7$ to $13 \%$ and -5 to $21 \%)$.
Figure 5 shows dose-response effects of the herbal medicine on blood coagulation when added in dispersion. It appears that the hemostatic effects increased with the dose as indicated by R. In addition to the similar effects on $\mathrm{R}$ and $\alpha$ as its powder form, the herbal material in dispersion also increased MA by $15 \%$, being more procoagulant than its powder form which had a minimal effect on MA.

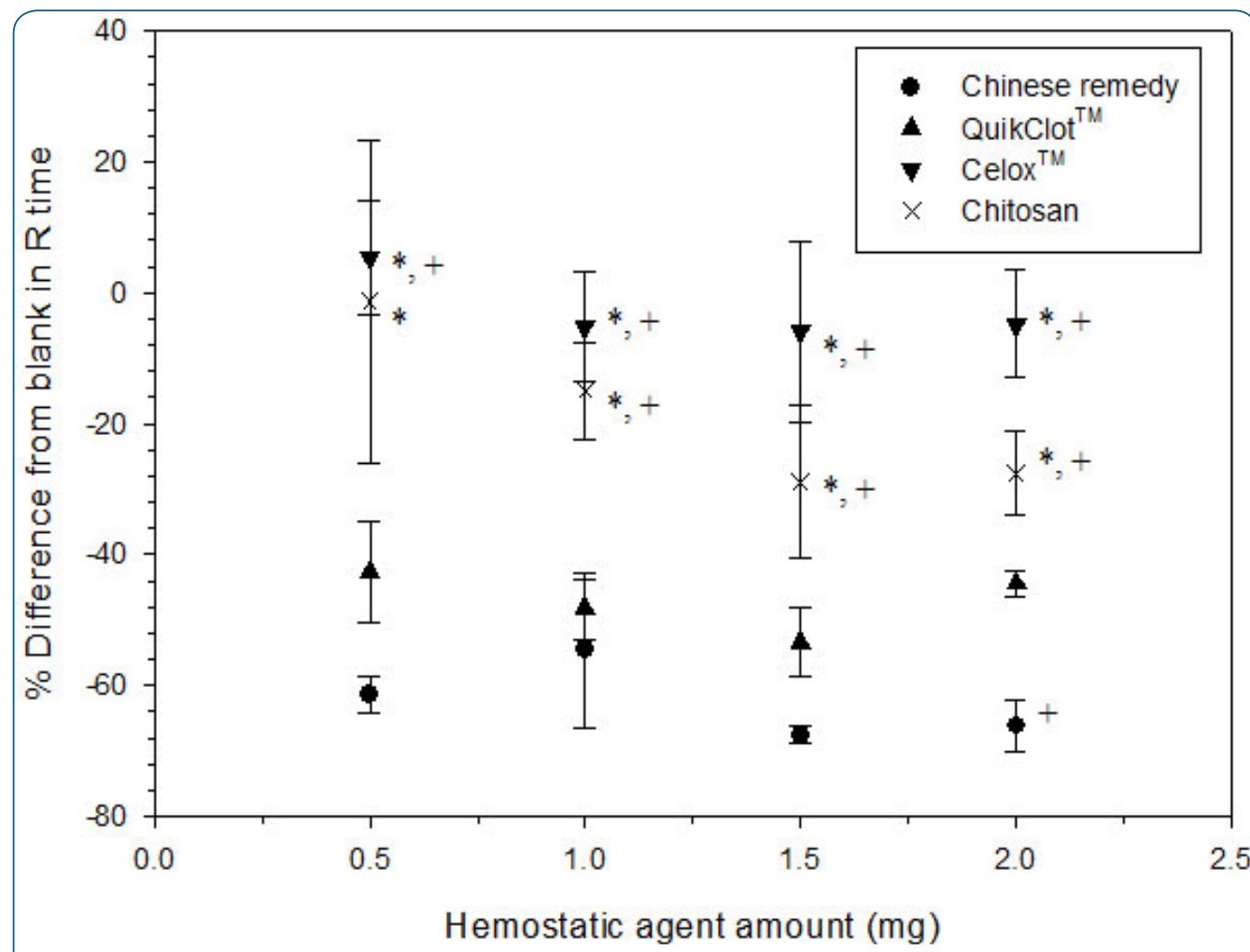

Figure 2: Effects of the amount of hemostatic agents added to blood on clot initiation time R. Data are expressed as mean \pm SD $(n=3 \sim 6) .^{*},+$ Different from Chinese remedy and QuikClot ${ }^{\mathrm{TM}}$, respectively $(\mathrm{p}<0.05)$

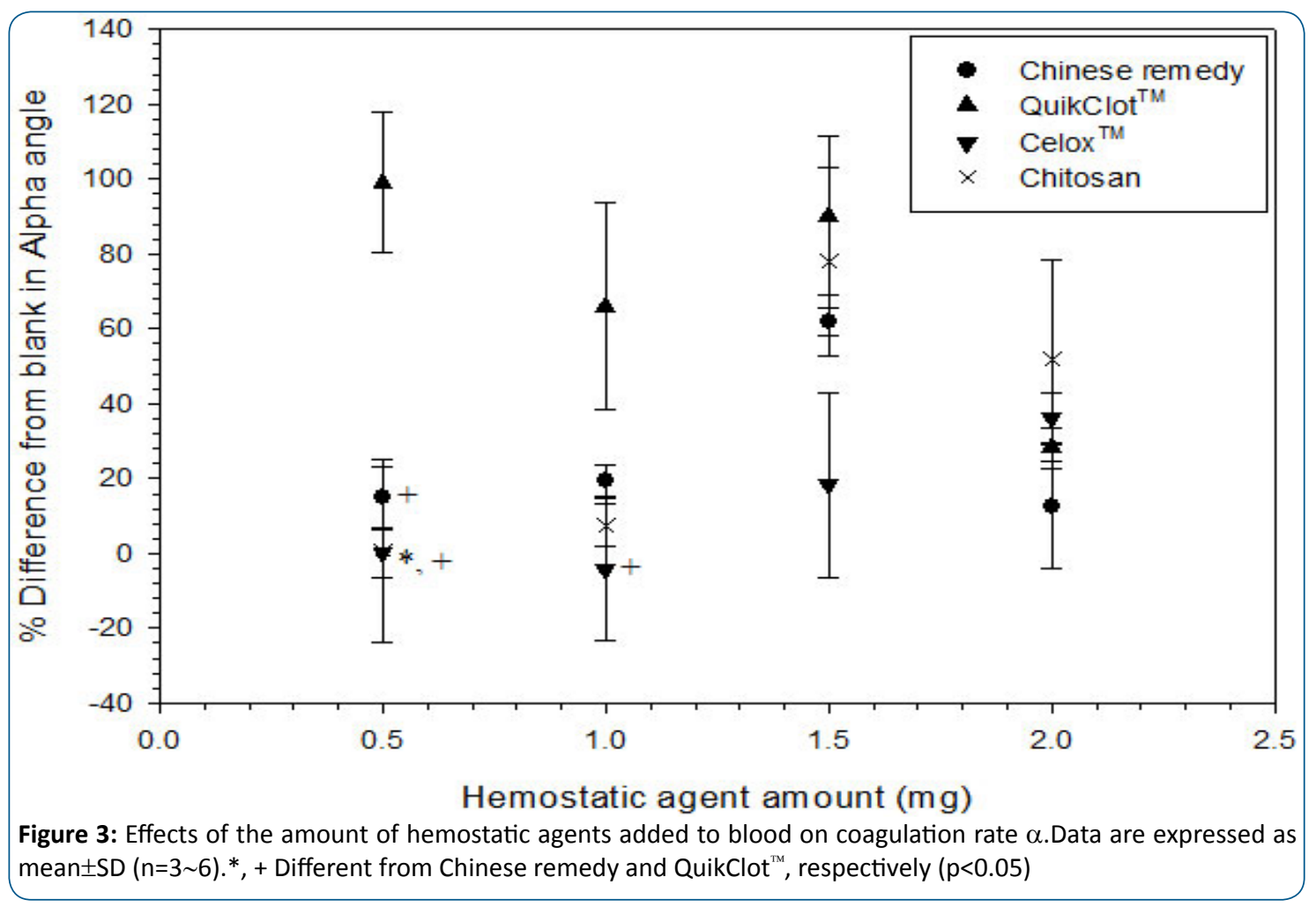


Citation: Henry T. Peng (2016) Comparative Study of Hemostatic Agents Using Thrombelastography. Mater. Sci. Eng. Adv. Res 1(4) : 1-7. doi: https://doi.org/10.24218/msear.2015.16.

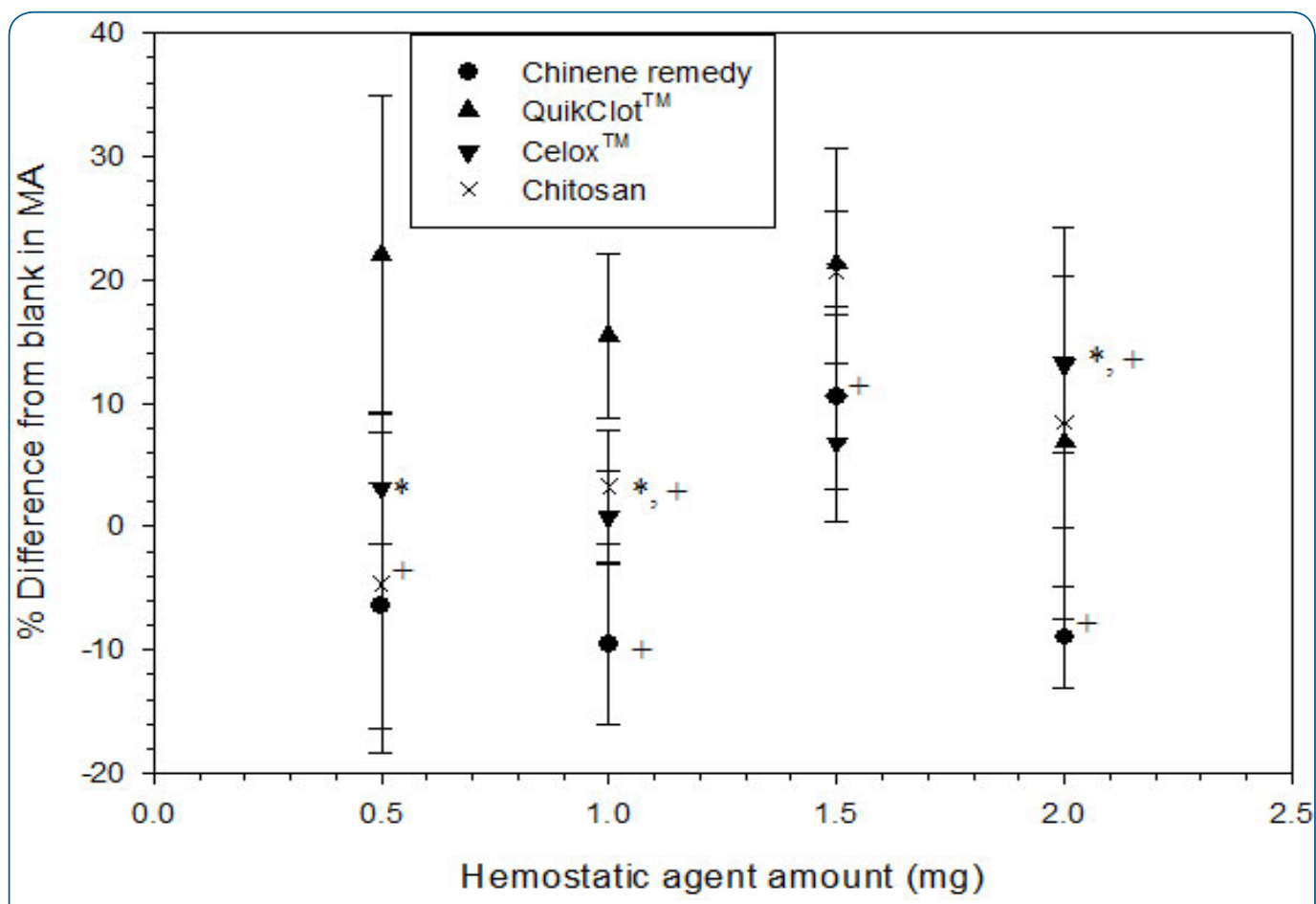

Figure 4: Effects of the amount of hemostatic agents added to blood on clot strength MA.Data are expressed as mean \pm SD $(n=3 \sim 6) .{ }^{*},+$ Different from Chinese remedy andQuikClot ${ }^{\mathrm{TM}}$, respectively $(\mathrm{p}<0.05)$

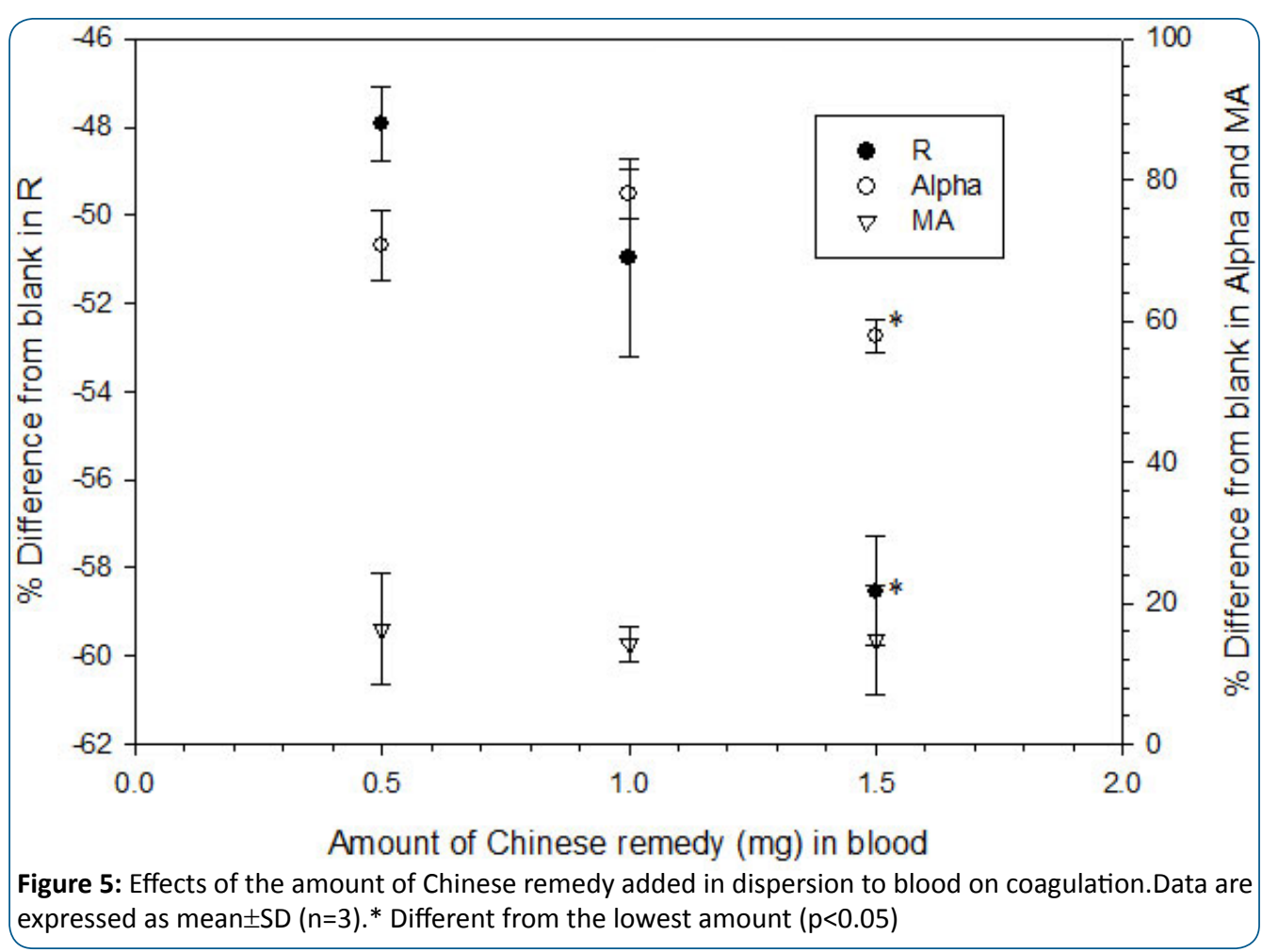

Figure 6 compares the effects of the hemostatic agents on the blood coagulation when added as dispersion in a buffer. The herbal remedy showed the most procoagulant effects as measured by reduced $\mathrm{R}$ time by $51 \%$, increased rate of clot formationa by $78 \%$ and increased maximum clot strength MA by $14 \%$, significantly larger than the corresponding values for QuikClot ${ }^{\mathrm{TM}}(33 \%, 14 \%$ and $-10 \%)$, Celox ${ }^{\mathrm{TM}}(0 \%,-7 \%$ and $0 \%)$ and chitosan $(33 \%,-19 \%$ and $-13 \%)$. Compared to their powder forms, all hemostatic agents except the herbal material became less procoagulant in the dispersion form.
Inaddition, Figure 7 comparesthecommercialhemostaticproducts (QuikClot ${ }^{\mathrm{TM}}$ and Celox ${ }^{\mathrm{TM}}$ ) withvarious chitosan biomaterials in powder forms. Specifically, chitosan powders were as effective as Celox $^{\mathrm{TM}}$ as indicated by the TEG parameters, but less effective than QuikClot ${ }^{\mathrm{TM}}$, which is in agreement with their hemostatic mechanism mainly by tissue adhesion.As far as the onset of clot formation $(\mathrm{R})$ is concerned, the block chitosan outperformed Celox $^{\mathrm{TM}}$, but not in terms of clot formation rate $(\alpha)$ and strength (MA). No significant differences were observed among the chitosan agents while block chitosan appears more effective than 

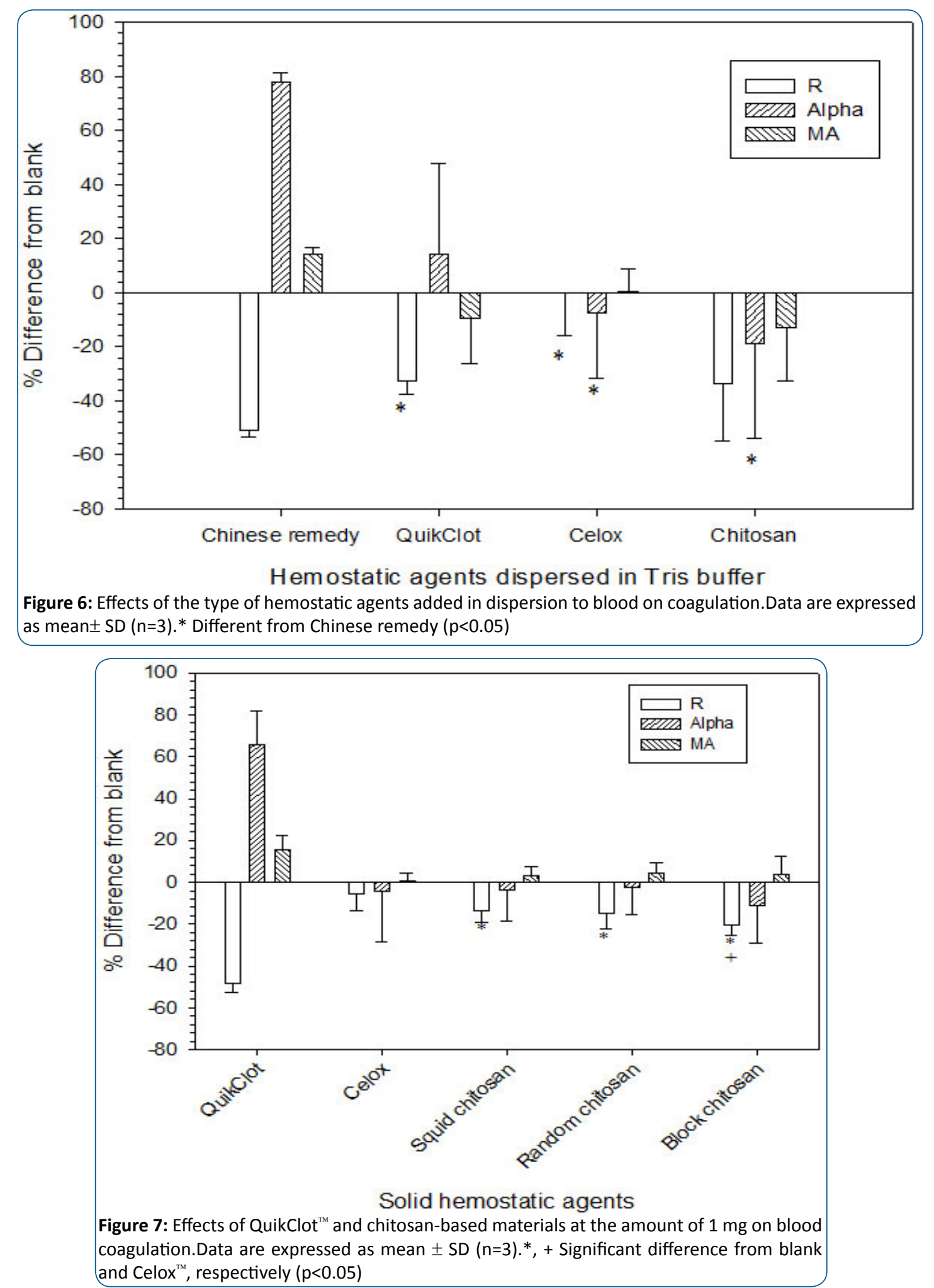

squid chitosan, likely due to their differences in chitosan source and physical structure. Furthermore, as implied by reduced R time (approximately 20\% decrease), all chitosan materials exhibited hemostatic activities, perhaps due to their induced formation of the coagulum of red blood cells and platelet aggregation as reported by many investigators [17].

\section{Discussion}

The hemostatic agents in this study are very different in their compositions and physiochemical properties and expected to have different hemostatic effects. This has been confirmed using TEG, a method that has been used to characterize various aluminosilicate materials for hemostasis [18]. It should be noted that the observed hemostatic effects of all agents, could not be attributed to an increase in blood viscosity, because in a control study where no calcium was added, no coagulation effects (i.e., no changes in blood viscosity) were observed in terms of $\mathrm{R}, \mathrm{a}$ and MA values.

Different herb medicines have been used for hemorrhage control such as notoginseng [19]. The observed effects of Yunnan Baiyao 
Citation: Henry T. Peng (2016) Comparative Study of Hemostatic Agents Using Thrombelastography. Mater. Sci. Eng. Adv. Res 1(4) : 1-7. doi: https://doi.org/10.24218/msear.2015.16.

on TEG variables are consistent with shortened bleeding and clotting time as measured by various techniques in vitro and vivo [20]. Compared to other commercial hemostatic materials in powder forms, the herbal material outperformed Celox ${ }^{\mathrm{TM}}$ and chitosan as indicated by all three TEG parameters. As far as the clotting time $(\mathrm{R})$ is concerned, the herbal material appears more effective than QuikClot ${ }^{\mathrm{TM}}$, but less effective in terms of clot formation rate ( $\alpha$ ) and strength (MA).

It is interesting that the formulation of the herbal remedy (dry powder vs. dispersion) affects its hemostatic properties. The initial material appeared as a homogenous powder, however, it contained granular particles of various sizes and clusters at a microscopic level. When dispersed in water the opaque conglomerated particles became singular and transparent [9]. Nanofibers with unique adhesive and structural properties were identified in the filtrate of its aqueous mixture and considered to play a role in platelet aggregation, clotting and healing of wounds [21].

Several mechanisms for hemostasis induced by the herbal material have been reported. Activation of blood platelets was observed to cause the release of platelet constituents and subsequent hemostatic effects [22]. Microchemical analysis has confirmed the herb medicine contains the preponderance of polysaccharide structure and significant amount of calcium [9]. The main active components in Yunnan Baiyao are saponins [23] although a total of 34 compounds were recently identified using liquid chromatography hybrid ion trap time-of-flight mass spectrometry [24]. Further identification of active ingredients in this herbal remedy and investigation of their hemostatic mechanisms are warranted to develop a superior hemostatic agent. Chitosan has well-documented bioactivities, such as antibacterial [25] and hemostatic properties [26,27,17]. The hemostatic potential of chitosan has long been recognized [27,28], however, its hemostatic potency reported in the literature varies significantly which could be due to the differences in physiochemical properties [17] as confirmed in this study.

Various mechanisms may contribute to its hemostatic properties and be postulated to be via vasoconstriction and the rapid mobilization of red blood cells, clotting factors, and platelet to the site of the injury, but the exact hemostatic mechanism of chitosan is still under study [26]. In one study where chitosan $(\mathrm{Mw}=80,000$, deacetylation degree $>80 \%)$ particle with 2.8 and 6.2 $\mathrm{mm}$ suspended in phosphate-buffered solution (PBS, $\mathrm{pH} 7.2$ ) at a concentration of $30 \mathrm{mg} / \mathrm{ml}$ reduced blood coagulation time and enhanced platelet aggregation [26]. Another study showed that chitosan $(\mathrm{Mw}=50,000$, deacetylation degree $>90 \%)$ significantly promoted platelet adhesion and aggregation through enhancing expression of platelet glycoprotein IIb/IIIa complex on platelet membrane surfaces and increasing intracellular calcium level in platelets [29]. Scanning and transmission electron microscopy revealed that red blood cells bound to chitosan particles and became cup-shaped [30]. Similar to QuikClot ${ }^{\mathrm{TM}}$, chitosan can also promote blood coagulation by its hydration leading to concentrated coagulation factors.A recent study showed that the hemostatic efficacy of chitosan was associated to its water binding capacity [31].

\section{Conclusion}

Different hemostatic agents were successfully evaluated and compared using TEG. When tested in a dispersion form, which may be easier to use (as a spray) than in a powder form, the herbal material provided better hemostasis than the other materials, where the coagulation actions of the latter were reduced in the dispersion form. The sources and methods for chitosan preparation could affect its hemostatic properties. The observed procoagulant effects of the hemostatic agentscould be attributed to different physiochemical and biological mechanisms. Further investigations of the herbal material and chitosan may be useful to pursue a potentially better substance for hemorrhage control.

\section{References}

1. Klages M, Zacharowski K, Weber CF. Coagulation management in trauma-associated coagulopathy: allogenic blood products versus coagulation factor concentrates in trauma care. Curr Opin Anaesthesiol. 2016; 29(2):245-249.doi: 10.1097/ ACO.0000000000000304.

2. Peng T. Biomaterials for Hemorrhage Control. Trends Biomater. Artif. Organs. 2010; 24(1):27-68.

3. Neuffer MC, McDivitt J, Rose D, King K, Cioonan CC, Vayer JS. Hemostatic dressings for the first responder: A review. Mil Med. 2004; 169(9):716-720.

4. Devlin JJ, Kircher S, Kozen BG, Littlejohn LF, Johnson AS. Comparison of ChitoFlex ${ }^{\circ}$, CELOX $^{\mathrm{TM}}$, and QuikClot ${ }^{\circ}$ in Control of Hemorrhage. J Emerg Med. 2011; 41(3):237-45. doi: 10.1016/j. jemermed.2009.02.017.

5. Wright JK, Kalns J, Wolf EA, Traweek F, Schwarz S, Loeffler CK, et al. Thermal injury resulting from application of a granular mineral hemostatic agent. J Trauma. 2004; 57(2):224-230.

6. Tang ZL, Wang X, Yi B, Li ZL, Liang C, Wang XX. Effects of the preoperative administration of Yunnan Baiyao capsules on intraoperative blood loss in bimaxillary orthognathic surgery: A prospective, randomized, double-blind, placebo-controlled study. Int J Oral Maxillofac Surg. 2009; 38(3):261-266. doi: 10.1016/j. ijom.2008.12.003.

7. Ladas EJ, Karlik JB, Rooney D, Taromina K, Ndao DH, Granowetter $L$, et al. Topical Yunnan Baiyao administration as an adjunctive therapy for bleeding complications in adolescents with advanced cancer. Support Care Cancer. 2012; 20(12):3379-3383. doi: 10.1007/ s00520-012-1598-1.

8. Yang $B, X u Z Q$, Zhang $H, X u F Y$, Shi XY, Zou Z, et al. The efficacy of Yunnan baiyao on haemostasis and antiulcer: a systematic review and meta-analysis of randomized controlled trials. Int J Clin Exp Med. 2014; 7(3):461-482.

9. Polesuk J, Amodeo JM, Ma TS. Microchemical investigation of medicinal plants. X - Analysis of the chinese herbal drug yunnan bai yao. Mikrochimica Acta. 1973; 61(4):507-517.

10. Carroll RC, Craft RM, Langdon RJ, Clanton CR, Snider CC, Wellons $\mathrm{DD}$, et al. Early evaluation of acute traumatic coagulopathy by thrombelastography. TransI Res. 2009; 154(1):34-39. doi: 10.1016/j. trsl.2009.04.001.

11. Schreiber MA, Differding J, Thorborg P, Mayberry JC, Mullins RJ, Timberlake GA, et al. Hypercoagulability is most prevalent early after injury and in female patients. J Trauma. 2005; 58(3):475-481. 
Citation: Henry T. Peng (2016) Comparative Study of Hemostatic Agents Using Thrombelastography. Mater. Sci. Eng. Adv. Res 1(4) : 1-7. doi: https://doi.org/10.24218/msear.2015.16.

12. Brazzel C. Thromboelastography-guided transfusion Therapy in the trauma patient. AANA J. 2013; 81(2):127-132.

13. Peng HT. Thromboelastographic study of biomaterials. J Biomed Mater Res B Appl Biomater. 2010; 94(2):469-485.doi: 10.1002/ jbm.b.31626.

14. Vig S, Chitolie A, Bevan DH, Halliday A, Dormandy J. Thromboelastography: a reliable test? Blood Coagul Fibrinolysis. 2001; 12(7):555-561.

15. Salooja N, Perry DJ. Thrombelastography. Blood Coagul Fibrinolysis. 2001; 12(5):327-337.

16. Pusateri AE, Delgado AV, Dick Jr EJ, Martinez RS, Holcomb JB, Ryan KL. Application of a granular mineral-based hemostatic agent (QuikClot) to reduce blood loss after grade $\mathrm{V}$ liver injury in swine. J Trauma. 2004; 57(3):555-562.

17. Whang HS, Kirsch W, Zhu YH, Yang CZ, Hudson SM. Hemostatic agents derived from chitin and chitosan. JMacromol Sci Polymer Rev. 2005; 45:309-323.

18. Baker SE, Sawvel AM, Zheng N, Stucky GD. Controlling bioprocesses with inorganic surfaces: Layered clay hemostatic agents. Chemistry of Materials. 2007; 19(18):4390-4392.

19. White CM, Fan C, Chow M. An Evaluation of the Hemostatic Effect of Externally Applied Notoginseng and Notoginseng Total Saponins. J Clin Pharmacol. 2000; 40(10):1150-1153.

20. Ogle CW, Dai S, Ma JC. The haemostatic effects of the Chinese herbal drug Yunnan Bai Yao: a pilot study. Am J Chin Med (Gard City N Y). 1976; 4(2):147-152.

21. Lenaghan SC, Xia L, Zhang M. Identification of nanofibers in the Chinese herbal medicine: Yunnan Baiyao. J Biomed Nanotechnol. 2009; 5(5):472-476.

22. Chew EC. Effects of Yunnan Bai Yao on Blood Platelets: An Ultrastructural Study. Comp Med East West. 1977; 5(2):169-175.
23. Liu XX, Wang L, Chen XQ, Deng XT, Cao $Y$, WangQ. Simultaneous quantification of both triterpenoid and steroidal saponins in various Yunnan Baiyao preparations using HPLC-UV and HPLC-MS. J Sep Sci. 2008; 31(22):3834-3846.doi: 10.1002/jssc. 200800382.

24. Dai C, Liang Y, Hao H, ZhengX, Xie L, Guan T, et al. Global detection and identification of components from Yunnan Baiyao based on liquid chromatography hybrid ion trap time-of-flight mass spectrometry. J Sep Sci. 2013; 36(12):1935-1944.

25. Rabea EI, Badawy MET, Stevens CV, Smagghe G, Steurbaut W. Chitosan as Antimicrobial Agent: Applications and Mode of Action. Biomacromolecules. 2003; 4(6):1457-1465.

26. Okamoto $Y$, Yano R, Miyatake K, Tomohiro I, Shigemasa $Y$, Minami S. Effects of chitin and chitosan on blood coagulation. Carbohydrate Polymers. 2003; 53(3):337-342.doi:10.1016/S01448617(03)00076-6.

27. Rao SB, Sharma CP. Use of chitosan as a biomaterial: Studies on its safety and hemostatic potential. J Biomed Mater Res. 1997; 34(1):21-28.

28. Arand AG, Sawaya R. Intraoperative chemical hemostasis in neurosurgery. Neurosurgery. 1986; 18(2):223-233.

29. Chou TC, Fu E, Wu CJ, Yeh JH. Chitosan enhances platelet adhesion and aggregation. Biochem Biophys Res Commun. 2003; 302(3):480483.

30. Smith CJ, Vournakis JN, Demcheva M, Fischer TH. Differential effect of materials for surface hemostasis on red blood cell morphology. Microsc Res Tech. 2008; 71(10):721-729.doi: 10.1002/jemt.20612.

31. Wu S, Huang Z, Yue J, Liu D, Wang T, Ezanno P, et al. The efficient hemostatic effect of Antarctic krill chitosan is related to its hydration property. Carbohydr Polym. 2015; 132:295-303. doi: 10.1016/j. carbpol.2015.06.030. 